\title{
A Method for Layered Identification of Defects in Trees Using Ground Penetrating Radar
}

\author{
Xiayang Xiao, Jian Wen*, Lin Gao, Zhongliang Xiao and Can Li \\ School of Technology, Beijing Forestry University, Beijing 100083, China E- \\ mail \\ Corresponding Author: Jian Wen, E-mail: WenJian@bjfu.edu.cn
}

\begin{abstract}
This paper addresses a method using ground-penetrating radar (GPR) to identify the defects (decays, cavities) and make layered identification in the trees. The method is able to make layered and present the trunk cross-section utilizing GPR and threshold layer technology. The interior defects of trees can be detected in early stage by using the non-invasive identification technology when the trees grow in the farm. To ensure the healthy growth of trees and avoid unnecessary loss, conservation measures should be adopted. The GPR technique offers a real-time and efficient non-invasive method in detecting the inner anomaly of tree. In this research, the tree was scanned at a selected elevation by moving a single radar unit along the bark tree in a circumferential manner. Then, the scanned data were handled to recognize the defective part of the tree by introducing threshold. Three logs with different features respectively were tested for verifying it. The error rate for identifying the defects in the depth and area that can be controlled within $20 \%$ and $15 \%$ respectively. In the future, with the improvement of detection and layered precision, the GPR can be a promising technique in the sphere of tree detection.
\end{abstract}

Keywords: GPR; layered identification; woods; decay; threshold

\section{Introduction}

There have been considerable researchers in the sphere of Nondestructive Testing (NDT) of wood for detecting internal defects, such as, Ocular Estimate, Stress Wave Method, Tree Needle Detection, Ultrasonic Technique, Computerized Tomography, and Radar Non-destructive Testing. While semi-and fully-destructive methods offer the most conclusive results, they are inappropriate for continual monitoring. Most methods cause a number of defects. Stress Wave Method needs pin nails in each measurement point. Tree Needle Detection required that probe is plunged into trees. These methods exhibit some damages for measured trees and make the measurement process cumbersome. In addition, Ultrasonic detection technique is vulnerable to outside interference such as equipment, coupling conditions and other factors, especially the problem caused by sensors connecting with materials. Computerized Tomography often costs high and security is also a key issue for CT using radioactive elements; a bad consequence may occur by misuse. Compared to the methods described above, Radar Testing is a non-destructive testing way for trees in true sense [1]. It has various advantages, such as non-destructive, non-pollution, sustainable, and security.

Ground penetrating radar (GPR) is a nondestructive detection technology using electromagnetic waves to detect the electrical characteristics of the medium, widely used in the field of shallow exploration [2], such as civil engineering [3], hydraulic engineering, field archeology and other fields [4]. In recent years, scholars also gradually take GPR for trees detection seriously. Studies pointed out that these anomalies could be associated the inner discontinuities between the bark and the 
internal trunk and lower velocities correspond to living trees, while higher velocities are associated with timber [5]. Other studies located the position of internal defects and the algorithm for identifying the defects in wooden logs using GPR [6]; some others showed relations between relative permittivity and moisture content of the different wood samples [7]. In addition, some make evaluation of wood beams in buildings based on GPR [7-8].

However, at present, most studies limit to the location of defects of trees and characteristics of electromagnetic wave propagation mechanism in trees. In the paper, firstly, it describes the principle of tree layering. Secondly, different reactions in different electromagnetic properties related to the GPR response are observed. Thirdly, it demonstrates the layered identification of the defects in the tree and generates visual image (simulation image of tree interior decay). Finally, it performs a full validation of potential applications and accuracy of GPR in the sphere of detection of trees through data analysis.

\section{GPR Principle}

\subsection{GPR Principle}

GPR is a geophysical survey methodology based on the transmission to the medium and a later reception of an electromagnetic wave. GPR emits high-frequency electromagnetic waves in the form of pulses from the transmitter of the antenna into the structure or material. These pulses have different refraction and reflection encountering different medium. When the pulses encounter subsurface interfaces or boundaries with different dielectric constants, the reflections are different. The internal defects have a different dielectric constant from that of surrounding wood [5]. Thus, the research is able to deduce the location of the internal defects of tree and make the defects layered recognition based on the properties. GPR transmits pulse by antenna $T$ and receives by receiver of antenna $\mathrm{R}$ (Figure 1 ).

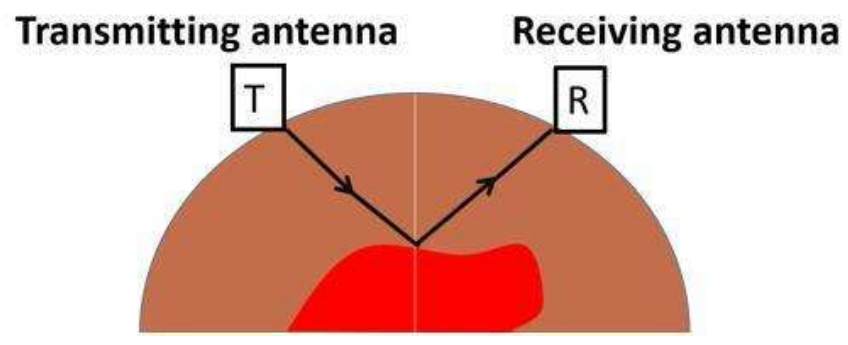

Figure 1. Principle for Radar Wave

Radar wave velocity $\left(\mathrm{V}_{\mathrm{p}}\right)$ is given as [1]:

$$
\mathrm{V}_{\mathrm{p}}=c / \sqrt{\varepsilon^{\prime}}
$$

The depth $(Z)$ from decay to interface is given as [2]:

$$
Z=\frac{c \times t}{2 \times \sqrt{\varepsilon^{\prime}}}
$$

$\varepsilon^{\prime}$ the dielectric constant of the medium, ${ }^{c}$ the velocity of electromagnetic waves in vacuum $=3 \times 10^{8} \mathrm{~m} / \mathrm{s}, Z$ the depth of the target of interest $(e . g$. defects) and ${ }^{t}$ the round-trip time.

In the case of electromagnetic waves under normal incidence, the amplitude reflection coefficient $R$ is given by Equation (3), where the subscripts 1 and 2 
denote the first and second media at the interface ${ }^{\varepsilon_{1}}$ and ${ }^{\varepsilon_{2}}$ are the dielectric constants of the two media [9].

$$
\mathrm{R}=\frac{\sqrt{\varepsilon_{1}}-\sqrt{\varepsilon_{2}}}{\sqrt{\varepsilon_{1}}+\sqrt{\varepsilon_{2}}}
$$

According to Fresnel formula (3): (1) The difference of electromagnetic properties of the medium on both sides and the intensity of reflected wave is positively correlated, and the property of medium on both sides can be deduced by the amplitude of the reflected waves. (2) When waves transmit from low to high dielectric constant medium, the reflection coefficient is negative, that is, the amplitude of the reflected wave reverse. On the contrary, when waves transmit from high to low dielectric constant medium, the reflection coefficient is positive, that is, the amplitude of the reflected wave is in the same direction. For example, the amplitude of the reflected radar waves reverses from air into the trunk. In contrast, the amplitude of the reflected radar waves from cavity of tree is in the same direction with radar wave. The reflections from tree bark and the reflection from the hollow area are in opposite direction. Therefore, the amplitude and direction of radar reflected waves are the key basis for differentiating interfacial electronic properties of two sides.

\subsection{GPR System}

The GPR system applied for the research is Tree Radar Detection System (TreeRadar, Inc.). The system contains two separate devices. As shown in Figure 2 , two experimenters collected a circumferential scan using a $900 \mathrm{MHz}$ GPR antenna equipped with a survey wheel and a field computer that applies the display of digital waveform and data storage. In this experiment, Salix babylonica L were used as experimental subject. In the processing, height of the measured cross-section and the starting point was marked. Then, radar took 360 degrees scan around the circumference of the tree at the height. In the end, radar scanning switched to a single point scanning mode, the radar was placed in the front side of the trees, with the metal plate placed in the corresponding backside. In this way, most of radar waves were reflected when encountered a mental plate. Thus, the propagation velocity and the average dielectric constant of trees could be calculated by the two-way travel time in the known distance (diameter trees) [10].

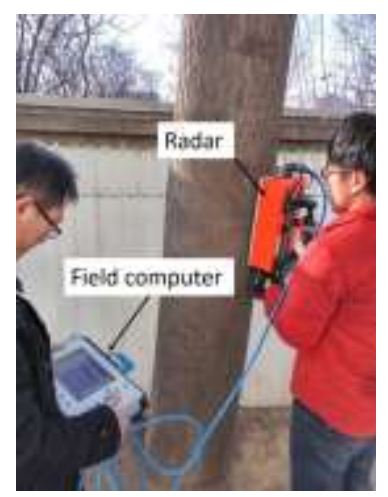

Figure 2. Data Acquisition 


\section{Data Analysis}

\subsection{Data Processing}

The purpose of processing of radar echo data was to remove noise, highlight the characteristic signal (reflected signal) and improve signal to noise ratio (SNR). Post-processing of the acquired data was necessary to make preparation for the detection of the internal defect position and horizon identification. The analysis software TGPR [11] that was independently developed was used data processing (Figure 3).

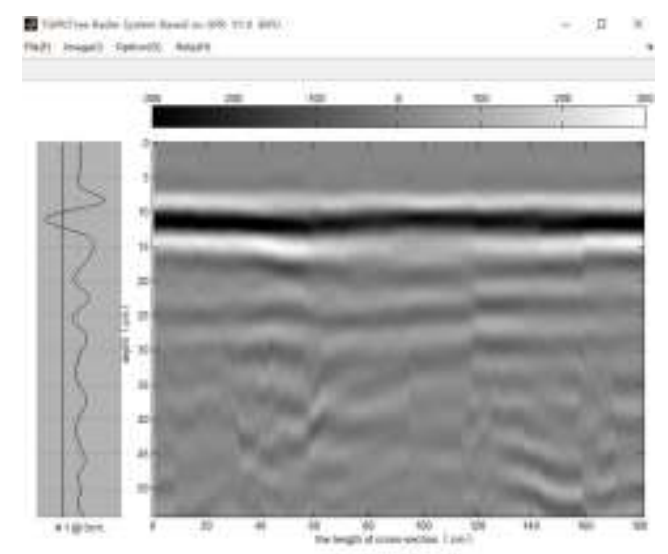

Figure 3. The Software Analysis System of Trees Based on GPR (TGPR)

The basic steps involved in post-processing were the application of range gain, zero correction and noise filtering [6].

\subsubsection{Range Gain}

Radar signals tend to attenuate the loss with increasing depth during the propagation of trees. The signal loss is greater at deeper detection area. Thus, the echo data require dependent gain that compensates for amplitude reduction with depth. Prior to compensate for gain, in order to ensure that the mean of data is zero, the A-Scan data must remove the DC component, avoiding the obvious zero drift. Signal gain processing requires data with less noise, or the noise may be magnified. In addition, the attenuation of radar signals is low near the surface but very high towards the center of the logs due to high moisture in the center. The TGPR software develops three types of gain: Standard Gain, Linear Gain and Gaussian Gain. In this research, the linear gain was mainly applied to radar data. After the gain was applied, the echo from the bottom of the log and some of the internal features became more obvious.

Attenuation model as follows [11]:

$$
A t)=C_{1} e^{-a t}+C_{2} e^{-a t}+\ldots+C_{n} e^{-a t t}
$$

\subsubsection{Zero Correction}

The time calculation of echo signals affects the accuracy of depth calculation. Therefore, zero calibration for positioning the bark of trees is necessary. The bark is calibrated to determine the sub-wave phase and polarity of echo signal. There are several possibilities for the position of primary wave of the tree bark (Figure 4). (1) primary wave locates the zero phases; (2) primary wave locates onequarter wavelength; (3) primary wave locates half wavelength; (4) primary wave 
locates three-quarters wavelength; (5) primary wave locates full wavelength. In the research, the primary wave located one-quarter wavelength. The contrasting images between the raw data and the data after zero correction are shown in Figure 5 (a) and Figure 5 (b).

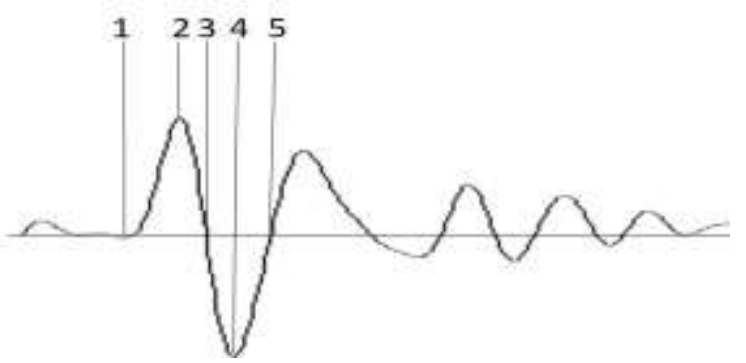

Figure 4. The Possibilities of Primary Wave

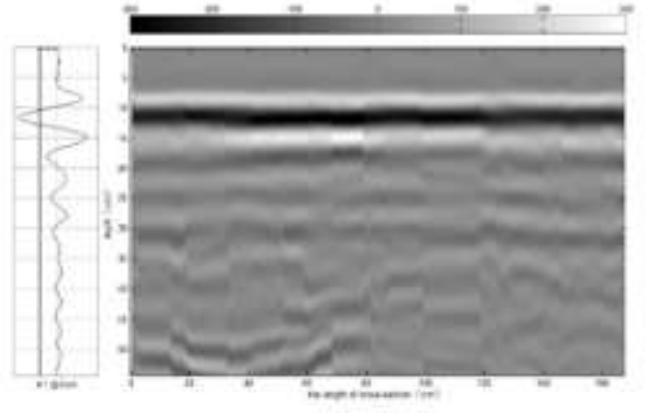

(a)

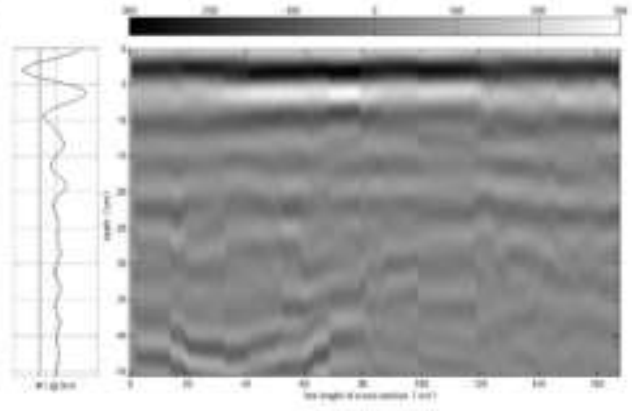

(b)

Figure 5. Comparison of Raw Data and Partial Processed Data

\subsubsection{Noise Filtering}

Noise affects the judgment of reflective surface seriously. The echo data of radar require noise filtering after zero correction. High-frequency noise, horizontal interference and direct coupling surface reflections need to be removed. The high frequency noise will result in "snow-like" noise in the data. When the range is set near the maximum limits for antenna or when large amount of gain is used, it often appears high-frequency noise.

Noise can be removed by vertical high-pass filter and vertical low-pass filter. The horizontal interference signal has a low frequency characteristic, with a vertical highpass filter corresponding treatment. The cut-off frequency of vertical high pass filter usually is set to about $1 / 3$ the center frequency of the antenna $(1 / 3 \times$ $900 \mathrm{MHz}=300 \mathrm{MHz})$. The low pass filter reduces the high frequency "snow-like" noise. The cut-off frequency of the vertical low pass filter is set at twice the center frequency of antenna $(2 \times 900 \mathrm{MHz}=1800 \mathrm{MHz})$. It is necessary to stress that filtering also has shortage of adding unwanted signals and removing desirable features from data. So one has to compare the unfiltered with filtered data during the analysis process. Moreover, adjust filter parameters as far as possible in the removal of radar clutter component of the original data and ambient noise signals, meanwhile, the echo signal without distortion. The above processing steps (amplitude gain, zero correction, noise filtering) were applied to all data. Subsequent chapters contain unprocessed raw data and the processed data [12]. 


\section{Experiment and Analysis}

\section{1. $\log 1$}

After the echo data were processed, trunk and the internal defects were predicted based on the signal changes. In Figure 6, the waveform of a single-channel data after zero calibration. The detection threshold is represented by dashed line. Echo reflected from the bark and decaying or hollow have been marked. The B-Scan figure of Log1 is shown in Figure 7. The blue line stands for the dots that are the locations of the biggest wave crest in every column. After doing a contrastive analysis, it is supposed that the location of the first biggest wave crest is where air/bark locates. Figure 8 (a) is one of the columns of echo signal figure which shows that radar wave signal detects the tree that is no decay and cavity. The detection threshold value (chosen as -40 in this paper) of decay or cavity is represented by the dashed line. The tree interior decay starts from the second wave trough, which exceeds the threshold. Hence, there are no abnormal phenomena (decay or cavity) inside log 1 . The reflection simulation picture of scanning $\mathrm{B}$ is illustrated in Figure 8 (b). In the end, the comparison and analysis between the detected result and the cut circular cross-sections can be seen from Figure 8(c) and (d).I

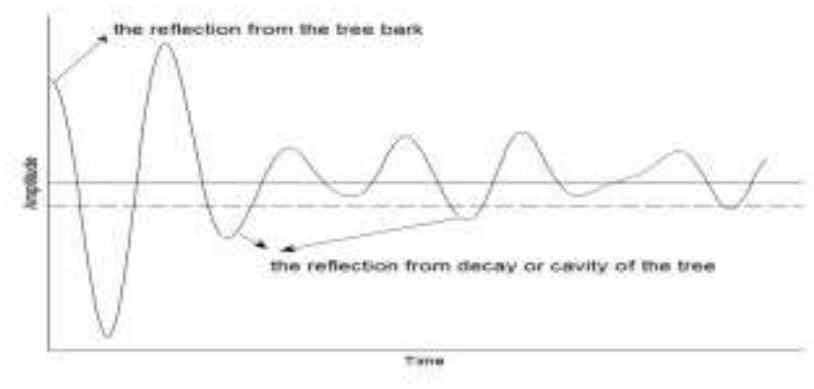

Figure 6. The Waveform of a Single-Channel Data after Zero Calibration

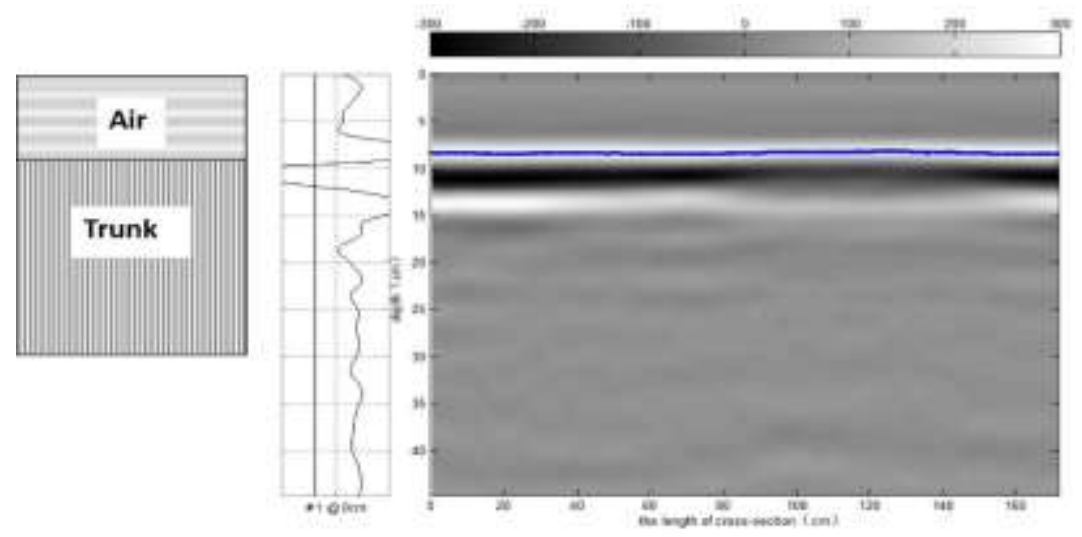

Figure 7. The B-SCAN Figure of Log 1 with No Decay or Empty 


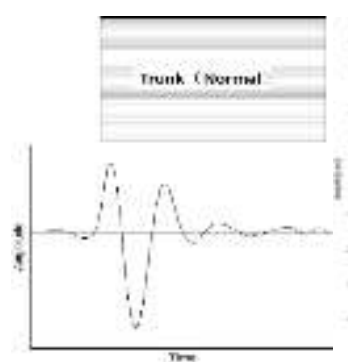

(a)

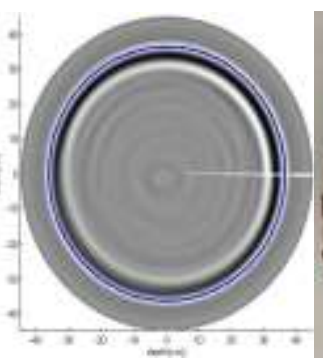

(b)

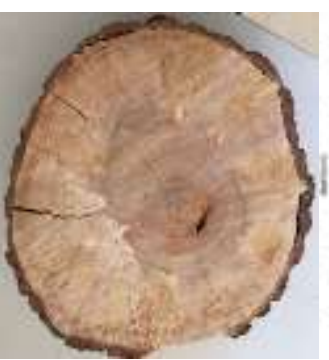

(c)

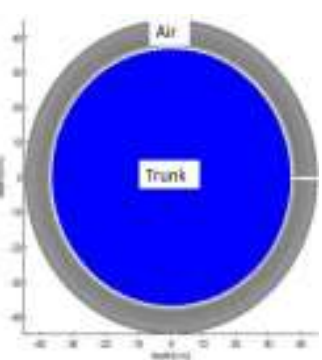

(d)

Figure 8. The Waveform of a Single-Channel and Cut Cross-Section of $\log 1$

\section{2. $\log 2$}

The magnitude of echo signal has a corresponding correlation with the change of medium inside the trees. According to the changes of signal, the defects inside the trees can be predicted. The red line (Figure 9) stands for the dots, which are locations of the second wave trough that exceeds the threshold in every column (Figure 6 the dashed line for threshold value). The line consists of red dots is the abnormal part of the trunk, which is likely to be the location of decay or cavity. The blue line is the location of trunk-air interface (Figure 9 and Figure 10(b)). The reactions of a column of the echo signal corresponding to air, trunk and abnormalities (decay or cavity) are shown in the Figure 10(a). Through analysis, the distance of A', B', C', D' and E' to the bark was $10.29 \mathrm{~cm}, 10.5 \mathrm{~cm}, 9.44 \mathrm{~cm}, 9.86 \mathrm{~cm}$ and $11.35 \mathrm{~cm}$, respectively (The average dielectric constant of $\log 2$ is 11 ). The $\log 2$ was sawed at positions where the processed data indicated changes in signal characteristics, which were attributed to presence of internal defects. The cut circular cross-sectional height could be measured and its corresponding estimating distance of A, B, C, D and E to that of bark was $10-15 \mathrm{~cm}$, $9.5 \mathrm{~cm}, 5.5-10 \mathrm{~cm}, 8-11.50 \mathrm{~cm}$ and $7-14.50 \mathrm{~cm}$, respectively in Figure $10(\mathrm{c})$. After the interpreted data is compared to the actual measurement data (both take the intermediate value), the measurement errors were $17.68 \%, 10.53 \%, 21.81 \%, 1.13 \%$ and $5.58 \%$ respectively (Table 1). In addition, calculated by using grid method, the actual error rate is $12.65 \%$ between the defect area S2 and the simulation defect area S2' (shown in Figure10(c) and Figure10 (d)). In conclusion, there is a good correlation between the changes in the signal characteristics and the actual location of defects observed after the sawing of log.
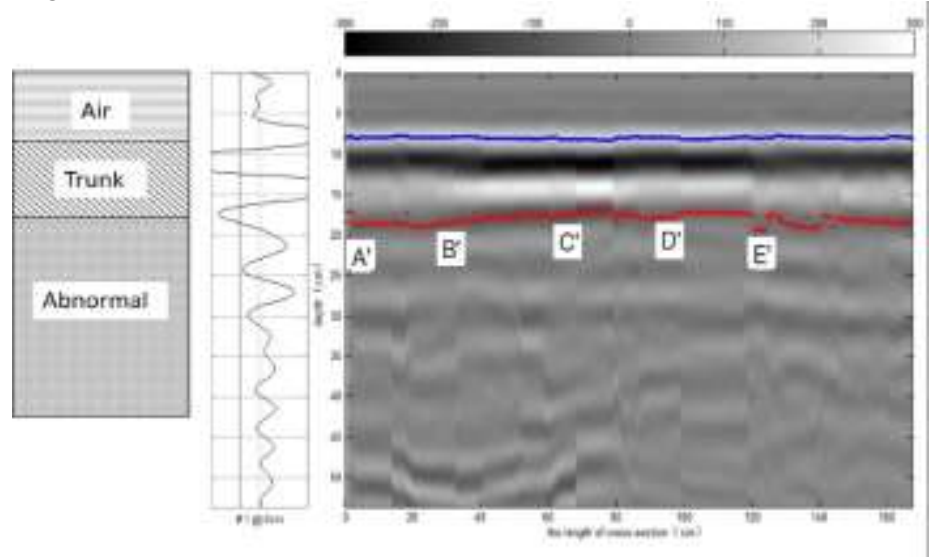

Figure 9. The B-SCAN Figure of Log2 with Decay or Empty 


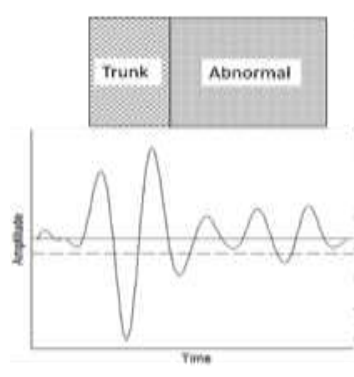

(a)

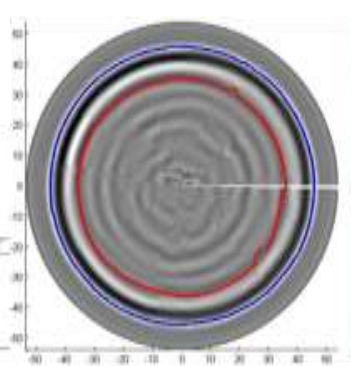

(b)

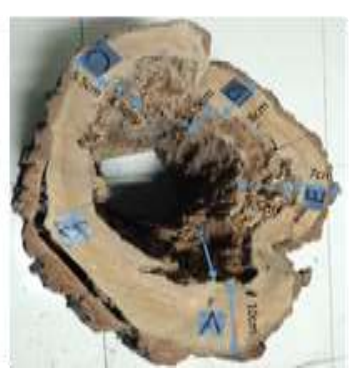

(c)

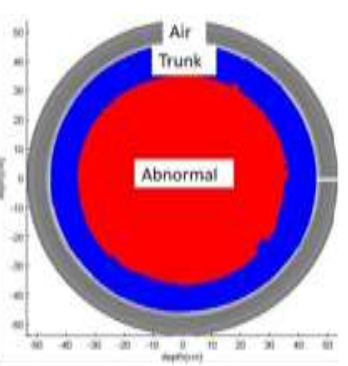

(d)

Figure10. The Waveform of a Single-Channel and Cut Cross-Section of $\log 2$

Table 1. Comparison of Test and Simulated Results for the Log 2 and Error Rate

\begin{tabular}{|c|c|c|c|c|c|c|}
\hline \multicolumn{3}{|c|}{ Actual location } & \multicolumn{4}{|c|}{$\begin{array}{lll}\text { Predicted location using } \\
\text { GPR }\end{array}$} \\
\hline $\begin{array}{l}\text { Defect } \\
\text { no. }\end{array}$ & Depth(cm) & $\operatorname{Area}\left(\mathrm{cm}^{2}\right)$ & $\begin{array}{l}\text { Defect } \\
\text { no. }\end{array}$ & $\operatorname{Depth}(\mathrm{cm})$ & Area $\left(\mathrm{cm}^{2}\right)$ & $\begin{array}{l}\text { Error rate } \\
\qquad \%)\end{array}$ \\
\hline $\mathrm{A}$ & $10-15$ & & $\mathrm{~A}^{\prime}$ & 10.29 & & 17.68 \\
\hline B & 9.5 & & $B^{\prime}$ & 10,50 & & 10.53 \\
\hline $\mathrm{C}$ & $5.5-10$ & & $\mathrm{C}^{\prime}$ & 9.44 & & 21.81 \\
\hline D & $8-11.5$ & & $\mathrm{D}^{\prime}$ & 9.86 & & 1.13 \\
\hline $\mathrm{E}$ & $7-14.5$ & & $E^{\prime}$ & 11.35 & & 5.58 \\
\hline S2 & & 502.15 & S2' & & 565.68 & 12.65 \\
\hline
\end{tabular}

\section{3. $\log 3$}

The Log 3 takes 13 as the average dielectric constant. Following the procedure similar to that for $\log 2$. The reactions of a column of the echo signal corresponding to air, trunk and abnormalities (decay or cavity) are shown in the Figure 11 and Figure 12(a). The blue line is the location of trunk-air interface (Figure 11 and Figure 12(b)). Through the experimental data, the distance of A', B', C' and D' to the bark was $10.09 \mathrm{~cm}, 9.25 \mathrm{~cm}$ and $16.73 \mathrm{~cm}$ respectively. After cutting down the trees, the crosssectional height can be measured and its corresponding estimating distance of $\mathrm{A}, \mathrm{B}, \mathrm{C}$ and $\mathrm{D}$ to that of bark was $10-12 \mathrm{~cm}, 10.50 \mathrm{~cm}, 9-10 \mathrm{~cm}$ and $15-17 \mathrm{~cm}$ respectively (Figure12(c)). Taking the experimental data compared to the actual measurement data, the measurement errors are $8.27 \%, 11.9 \%, 4.74 \%$ and $4.56 \%$ respectively. In addition, calculated by using grid method, the actual error rate is $9.52 \%$ (Table 2) between the defect area S3 and the simulation defect area S3' (shown Figure12 (d)). 


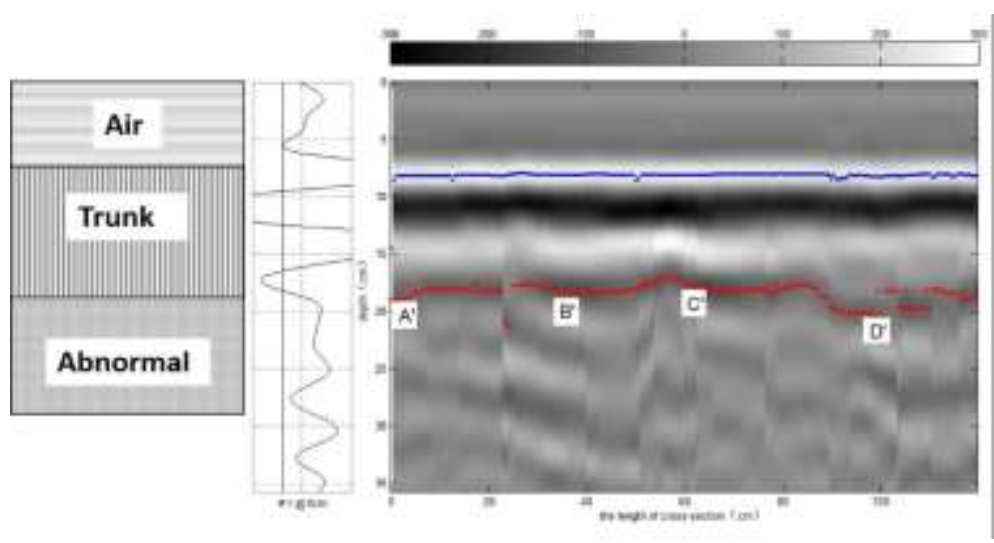

Figure 11. The B-SCAN Figure of Log3 with Decay or Empty

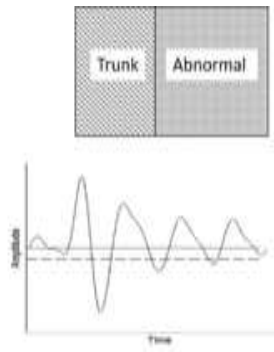

(a)

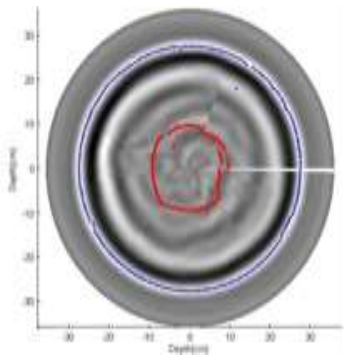

(b)

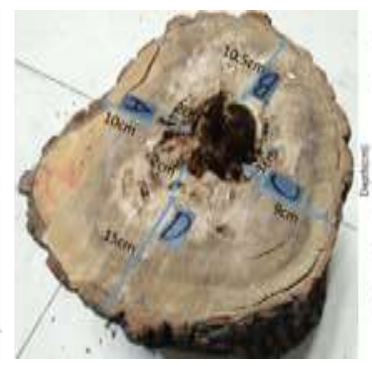

(c)

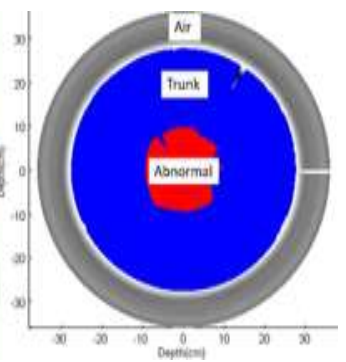

(d)

Figure 12. The Waveform of a Single-Channel and Cut Cross-Section of $\log 3$

Table 2. Comparison of Test and Simulated Results for the Log 3 and Error Rate

\begin{tabular}{|c|c|c|c|c|c|c|}
\hline \multicolumn{3}{|c|}{ Actual location } & \multicolumn{2}{|c|}{$\begin{array}{c}\text { Predicted location } \\
\text { using GPR }\end{array}$} & \multirow[b]{2}{*}{ Area $\left(\mathrm{cm}^{2}\right)$} & \multirow[b]{2}{*}{$\begin{array}{c}\text { Error } \\
\text { rate } \\
(\%)\end{array}$} \\
\hline $\begin{array}{c}\text { Defect } \\
\text { no. }\end{array}$ & Depth $(\mathrm{cm})$ & Area $\left(\mathrm{cm}^{2}\right)$ & $\begin{array}{c}\text { Defect } \\
\text { no. }\end{array}$ & $\operatorname{Depth}(\mathrm{cm})$ & & \\
\hline A & $10-12$ & & $A^{\prime}$ & 10.09 & & 8.27 \\
\hline B & 10.5 & & $\mathrm{~B}^{\prime}$ & 9.25 & & 11.9 \\
\hline C & $9-10$ & & $C^{\prime}$ & 9.05 & & 4.74 \\
\hline D & $15-17$ & & D' & 16.73 & & 4.56 \\
\hline S3 & & 109.24 & S3, & & 98.84 & 9.52 \\
\hline
\end{tabular}

\section{Conclusion and Suggestion}

This research applied ground-penetrating radar to detect the Salix babylonica $\mathrm{L}$ nondestructively. There is a very good correlation between the simulation of the trunk cross-section and those found after sawing the logs. The method of threshold value is introduced to identify and stratify the internal defects of the tree and the error rate in the depth and area can be controlled within $20 \%$ and $15 \%$ respectively. This study indicates that GPR layer technique in the detection of defects in trees can be achieved.

While this study has shown that GPR technique is feasible and effective in the sphere of layered identification of defects in the trees, there are many errors in the study. For example, the shapes of trees are irregular, which causes difficulty in 
the detection of errors in the tree size. Moreover, the average of dielectric constant is applied to compute depth in the experiment, but the dielectric constant of the trees is different for each measurement position actually. Then, the method of threshold value is relatively single on the layered identify metrics. Therefore, the accuracy of layered identification is not high.

Later these investigations will be made and the error rate will be decreased. To accurately measure the position of the dots laser radar or contour scanning method is applied, which can reduce the errors of size measurement. Besides, a modification is made in terms of dielectric constant of measured trees at every position. Then, Hilbert-Huang transform method is applied to multi-scale analyze (instantaneous frequency, instantaneous phase and instantaneous amplitude) the layered identification and judge the approximate location of the abnormality or hierarchy inside the trees. Based on the previous research on the localization of the defect in the trees, this essay will show more in-depth research on a hierarchical identification of the defect in the trees and presents a visual image of the defected layers inside the trees

\section{Acknowledgments}

This work was supported by "the Fundamental Research Funds for the Central Universities (NO. 2015ZCQ-GX-01, BLX2013008).

\section{References}

[1] L. Gao and J. Liu, "Non-destructive detection of standing trees and radar wave detection", 2010 International Conference on Computing, Control and Industrial Engineering, Wuhan, China, (2010).

[2] L. M. Collins and Peter Torrione, "Algorithms for landmine detection using the NIITEK ground penetrating radar", Detection and remediation technologies for mines and minelike targets VII, Proceedings of SPIE, vol. 4742, (2002), pp. 709-718.

[3] A. Benedetto and F. Benedetto, "Reliability of signal processing technique for pavement damages detection and classification using ground penetrating radar", IEEE Sensors Journal, vol. 5, no. 2, (2005), pp. 3,471-480.

[4] A. Benedetto, "A three dimensional approach for tracking cracks in bridges using GPR", Journal of Applied Geophysics, vol. 97, no.10, (2013), pp. 37-44.

[5] H. Lorenzo and V. Pérez-Gracia, "Forestry applications of ground-penetrating radar", Forest Systems, vol.19, no.1, (2010), pp. 5-17.

[6] U. B. Halabe and S. Agrawal, "Bhaskaran Gopalakrishnan. Nondestructive evaluation of wooden logs using ground penetrating radar", Nondestructive Testing and Evaluation, vol. 24, no. 4, (2009), pp. 329-346.

[7] S. Razafindratsima and F. Demontoux, "Non-destructive evaluation of timber structures using GPR technique", 15th International Conference on Ground Penetrating Radar, Brussels, Belgium, (2014).

[8] V. P. Gracia and S. S. Assun, "Study of wood beams in buildings with ground penetrating radar", 15th International Conference on Ground Penetrating Radar, Brussels, Belgium, (2014).

[9] U. B. Halabe, S. H. Petro and H. V. S. G. Rao, "Nondestructive evaluation methods for highway bridges superstructures", Constructed Facilities Center, Report No. CFC 95-215, Department of Civil and Environmental Engineering, West Virginia University, Morgantown, WV, (1995).

[10] J. R. Butnor and M. L. Pruyn, "Detecting defects in conifers with ground penetrating radar: applications and challenges", Forest Pathology, vol. 39, no. 5, (2009), pp. 309-322.

[11] A. Tzanis, "matGPR Release 2: A freeware MATLAB® package for the analysis \& interpretation of common and single offset GPR data, fastTIMES, vol. 15, no.1, (2010), pp. 17-43.

[12] D. Devaru, U. B. Halabe, "Algorithm for detecting defects in wooden logs using ground penetrating radar", Intelligent Systems in Design and Manufacturing VI, Proceedings of SPIE, vol. 5999, no .6, (2005), pp.110-121.

\section{Authors}

Xiayang Xiao, he graduated at the School of Technology, Beijing Forestry University.

Jian Wen, he got his Phd at the School of Technology, Beijing Forestry University. 